\title{
A Smart Health Monitoring Technology
}

\author{
Carl Chalmers ${ }^{1}$, William Hurst ${ }^{1}$, Michael Mackay ${ }^{1}$, Paul Fergus ${ }^{1}$ \\ ${ }^{1}$ Liverpool John Moores University, Byrom Street, Liverpool, L3 3AF, UK. \\ \{C.Chalmers, W.Hurst, M.I.Mackay, P.Fergus\} $l$ ljmu.ac.uk
}

\begin{abstract}
With the implementation of the Advanced Metering Infrastructure (AMI), comes the opportunity to gain valuable insights into an individual's daily habits, patterns and routines. A vital part of the AMI is the smart meter. It enables the monitoring of a consumer's electricity usage with a high degree of accuracy. Each device reports and records a consumer's energy usage readings at regular intervals. This facilitates the identification of emerging abnormal behaviours and trends, which can provide operative monitoring for people living alone with various health conditions. Through profiling, the detection of sudden changes in behaviour is made possible, based on the daily activities a patient is expected to undertake during a 24-hour period. As such, this paper presents the development of a system which detects accurately the granular differences in energy usage which are the result of a change in an individual's health state. Such a process provides accurate monitoring for people living with self-limiting conditions and enables an early intervention practice (EIP) when a patient's condition is deteriorating. The results in this paper focus on one particular behavioural trend, the detection of sleep disturbances; which is related to various illnesses, such as depression and Alzheimer's. The results demonstrate that it is possible to detect sleep pattern changes to an accuracy of $95.96 \%$ with 0.943 for sensitivity, 0.975 for specificity and an overall error of 0.040 when using the VPC Neural Network classifier. This type of behavioral detection can be used to provide a partial assessment of a patient's wellbeing.
\end{abstract}

Keywords: Smart Grids, Advanced Metering Infrastructure, Smart Meters, Profiling, Assistive Technologies, Early Intervention Practice, Customer Access Devices, Activates of Daily Living.

\section{Introduction}

Many countries around the world are currently undertaking a large-scale implementation of smart meters and their associated infrastructures. These smart energy devices offer vast advancements to the traditional energy grid. However, in order to maximise their true potential, different applications need to be considered beyond the traditional uses of electricity generation, distribution and consumption. The bidirectional communication capabilities provided by this smart technology is supported by the Advanced Metering Infrastructure (AMI), which forms a fundamental part of the smart grid.

The smart metering infrastructure provides new possibilities for a variety of different applications that where unachievable using the traditional grid topology. Specifically, smart meters, enable detailed around the clock monitoring of energy usage. This granular data captures detailed habits and routines through the users' 
interactions with electrical devices and appliances. Each smart meter accurately records the electrical load for a given property at 30-minute intervals. However, this reading frequency can be reduced to obtain even finer readings. This data enables the detection and identification of sudden changes in patient behaviour.

Each year, the number of people in the UK living with self-limiting conditions, such as dementia, Parkinson's disease and mental health problems, is increasing [1]. This is largely due to an aging population and improvements in diagnosis and medical treatments. The number of populace living with dementia worldwide [2] is currently estimated at 35.6 million and this number is set double by 2030 and more than triple by 2050 . Additionally, 1 in 4 people currently experience a type of mental health problem each year [3]. Supporting these sufferers places a considerable strain on organisations, such as the National Health Service (NHS), local councils, front line social services and carers / relatives [4]. In monetary terms, dementia alone costs the NHS over $£ 17$ billion a year [5]; exacerbated by the cost of depression patients, which is predicted to increase to 1.45 million in the UK; adding a further $£ 2.96$ billion cost to essential services by 2026 . This figure excludes other mental health conditions, such as Anxiety disorders, Schizophrenic disorders, bipolar related conditions, eating disorders and personality disorders.

Effective around the clock monitoring of these conditions can be a considerable challenge and often leads to patients having to reside in care homes and other accommodation. In addition, the need to detect accurately, sudden or worsening changes in a patient's condition, is vital for early intervention.

Community mental health groups, crisis and home resolution teams, assistive outreach teams and early psychosis teams all play a key role in preventing costly inpatient admissions. If any changes are not dealt with early, the prognosis is often worse and, as a result, costs for treatment will undoubtedly be higher [6]. An early intervention approach has been shown to reduce the severity of symptoms, improve relapse rates and significantly decrease the use of inpatient care. Evidence suggests that a comprehensive implementation of Early Intervention Practice (EIP) in England could save up to $£ 40$ million a year in psychosis services alone. Being able to detect deteriorating conditions in dementia patents earlier, enables physicians to better diagnose and identify stage progression for the disease. This enables earlier intervention for the illness before cognitive deficits affect or worsen mental capacity; supporting the individual and their family in adapting to the illness simultaneously.

This paper explores the idea of utilising smart meter data to detect abnormal changes in behaviour using a supervised classification approach. Section 2 provides a background into smart meters and their supporting technologies along with patient behaviours. Section 3 presents an insight into the behavioural trends that smart meters can detect at different reading intervals. Additionally, we present a case study using sample smart meter data to detect sleep disturbances. Section 4 discusses the proposed methodology and system framework, while section 5 presents the results. The paper is concluded in in section 6 . 


\section{Background}

Smart grids fundamentally change the way in which electricity is generated, distributed and monitored. This enhanced communication removes the traditional need for energy usage readings to be collected manually. Instead, a robust automatic reporting system with greater granularity of readings is offered [8].

\subsection{Advanced Metering Infrastructure}

The AMI provides bidirectional communication between the consumer and the rest of the smart grid. A smart grid is a complex modern electricity system [7]. It uses sensors, monitoring, communications, and automation, to improve the electricity system.

There are many advantages of deploying the AMI, some of which include: reduced costs for meter readings (possibility to access meters otherwise difficult to attend due to position or security reasons), support for real-time pricing, increased fraud detection and reduced read-to-bill time to name but a few. As part of the larger smart grid, the AMI can be broken down into three specific areas, each with their own roles and functions, as shown in Figure 1.

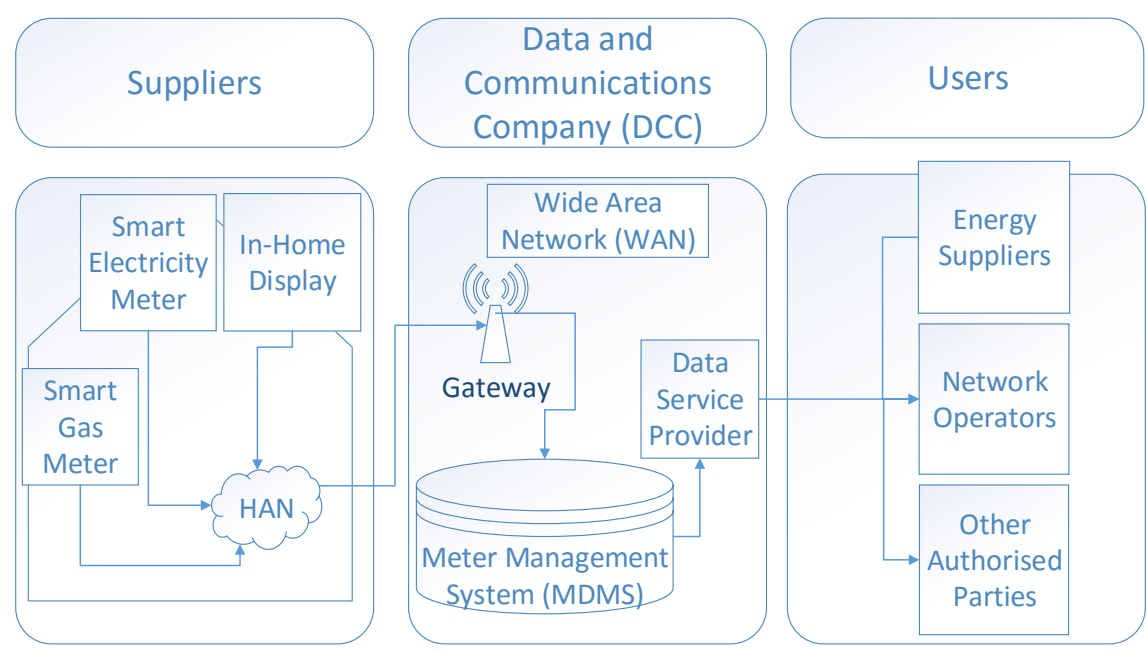

Figure 1. Advanced Metering Infrastructure (AMI).

The Home Area Network (HAN) is housed inside the consumer premises and is made up of a collection of different devices. Firstly, the In-Home Display unit (IHD), which is the most visible and accessible part of the AMI. It provides the consumer with up to date information, in real-time, on electricity usage, as well as the units of energy that are being consumed. Secondly, the smart meter provides real-time energy usage to both the consumer and all of the stakeholders. Smart meters are able store 13 months of data keeping a record of total energy consumption. In addition to smart meters, smart plugs enable the identification of the individual devices that are 
responsible for the reported energy usage. Additionally, they provided detailed information about their duration of use.

The Wide Area Network (WAN) controls the communication between the HAN and the utility companies. The WAN is responsible for sending all polled meter data to the utility companies and other grid stakeholders, using a robust backhaul network, such as Carrier Ethernet, GSM, CDMA or 3G [9]. The geographical location of the smart meter dictates what WAN technologies need to be implemented, this is due to the constraints of certain communication technologies. The Data Aggregator Unit (DAU) is the communication device that is used to collect the energy usage data form the home gateway or the smart meter. The acquired data is transmitted using one of the communication technologies mentioned above to the control centre. The Meter data management system MDMS is the central control centre which provides the storage and data processing capabilities for the obtained smart meter data. The MDMS also collects information regarding the status for the generation, transmission and distribution of the wider smart grid.

Service users are a number of organisations and utility companies that have access to the data for analysis purposes. Energy suppliers communicate remotely with the smart metering equipment in order to perform a number of tasks. This might include taking meter readings, updating price information on the in-home display and identifying readings on a change of supplier or change of tenancy. Consumers can allow other organisations to have access to the data from their smart meter.

\subsection{Smart Meters}

Smart meters the foundation of any future smart electricity grid and provide consumers with highly reliable, flexible and accurate metering services. Most significantly, they provide real-time energy usage readings at granular intervals [11]. They obtain information from the end users' load devices and measure the energy consumption. Added information, such as any home-generated electricity information, is provided to the utility company and/or system operator for better monitoring and billing process. Smart meters are able to store 13 months of historical energy usage, which allows for the creation of detailed energy usage profiles [12]. Currently readings are taken at 30 minute intervals. However, as these meters become more sophisticated, they are able to measure household power consumption at even more granular time-scales [13]. Smart meters are able to report energy usage as low as 10second intervals, even though this is not currently widely deployed due to the significant amount of data it would generate [14]. This is of particular benefit for health monitoring applications, where changes in behaviour need to be identified at the granular level. Figure 2 highlights, the additional information that can obtained from increasing the reading frequency. 
Information Obtained From Data

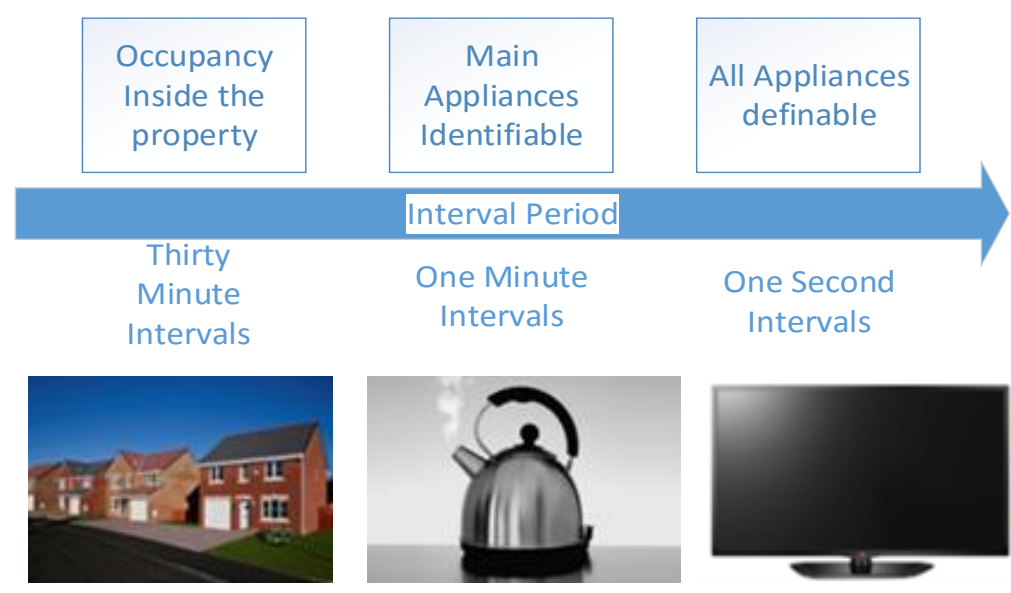

Figure 2. Information obtained by increasing interval reading.

Smart meters utilise ZigBee smart energy. The ZigBee Alliance forms a collection of device descriptions and functions that allow energy providers to manage and monitor energy loads to optimise consumption. Over one million ZigBee electric meters have been deployed by a variety of utility companies in the USA. The UK Department of Energy \& Climate Change (DECC) has announced Smart metering equipment technical specifications (SMETS) 2, which cites the use of ZigBee Smart Energy 1.x. In addition, this communication standard is able to collect energy usage reading at even more granular intervals overcoming the default 30-minute frequency set by the communication providers [15]. Consumer Access Devices (CAD), will access updated consumption and tariff information directly from their smart meter; a $\mathrm{CAD}$ can request updates of electricity information every 10 seconds and gas information every 30 minutes.

As previously, discussed, smart meter data enables active in-home monitoring. By analysing past behaviours, and improved prediction of worsening conditions is made possible. Analysing the data in this manner facilitates early intervention and an improved outcome for the patient by ensuring their medical and care needs are sufficient.

\subsection{Patient Behaviours}

Being able to detected and predict these changes in patterns of behaviour requires a detailed understanding of the symptoms that are anticipated for each medical condition.

For example, there are a common set of features for conditions such as Alzheimer's disease and other dementias. These include agitation, anxiety, depression, apathy, delusions, sleep and appetite disturbance, elation, irritability, disinhibition and hallucinations [16]. The severity of each of these conditions alters with disease 
progression. Likewise, severe depression exhibits many similar behavioural symptoms as dementia, e.g. memory problems and social disengagement. Additionally, depression can cause physical complications, such as chronic joint pain, limb pain, back pain, gastrointestinal problems, tiredness, sleep disturbances, psychomotor activity changes and appetite changes [17]. These changes are reflected in how the sufferer interacts with people, their environment and their electric devices. Being able to detect any erratic or sudden behaviour change early, caters for better intervention and can lead to an early diagnosis in psychosis. Each individual is different and, as such, present their own set of symptoms and warning signs; however, one or more warning signs are likely to be evident, for example:

- Memory problems.

- Severe distractibility.

- Severe decline of social relationships.

- Dropping out of activities - or out of life in general.

- Social withdrawal, isolation, reclusiveness.

- Odd or bizarre behaviour.

- Feeling refreshed after much less sleep than normal.

- Deterioration of personal hygiene.

- Hyperactivity or inactivity, or alternating between the two.

- Severe sleep disturbances.

- Significantly decreased activity.

Understanding these often subtle changes can lead to reduced hospital admissions through the implantation of an early intervention care plan. This is especially true for dementia suffers where the understanding of a patient's ability to undertake normal Activates of Daily Living (ADL) is an important component of the overall assessment of a patient's wellbeing. For that reason, the following section discusses how smart meter data can be utilised to detect specific patterns and device interactions.

\section{Approach}

In order to simulate the real-time data gathering capabilities of a smart meter, an energy monitor has been installed in a patient's premise to gather energy usage data. Energy usage is collected in real-time. This establishes the patient's routine and identifies any noteworthy trends in device utilisation.

\subsection{Case Study}

Figure 3 shows an overview of the electricity monitor, shown in (a). The blue current sensor transformer clip (CT) is fastened around the live cable shown in (b) to measure the electrical load. The second white sensor, which is the Optical Pulse Sensor, shown in (c) works by sensing the LED pulse output from the utility meter. 


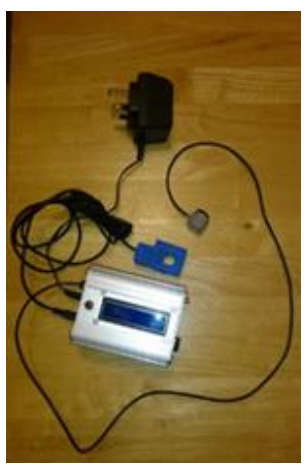

(a)

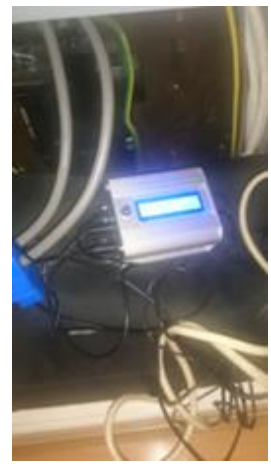

(b)

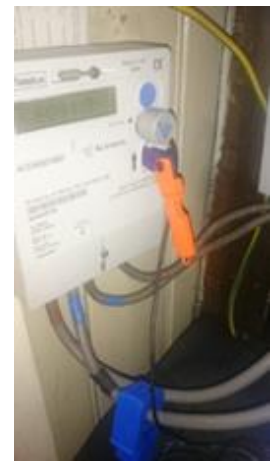

(c)

Figure 3. Energy monitor.

Each pulse corresponds to a certain amount of energy passing through the meter. By counting these pulses, a $\mathrm{kWh}$ value can be calculated. Due to smart meters not being generally available until 2020 energy monitors can be used to simulate smart meter data collection capabilities. The data obtained from the energy monitor is logged to an SQL database as demonstrated in Figure 4. The proposed system interfaces with the database directly.

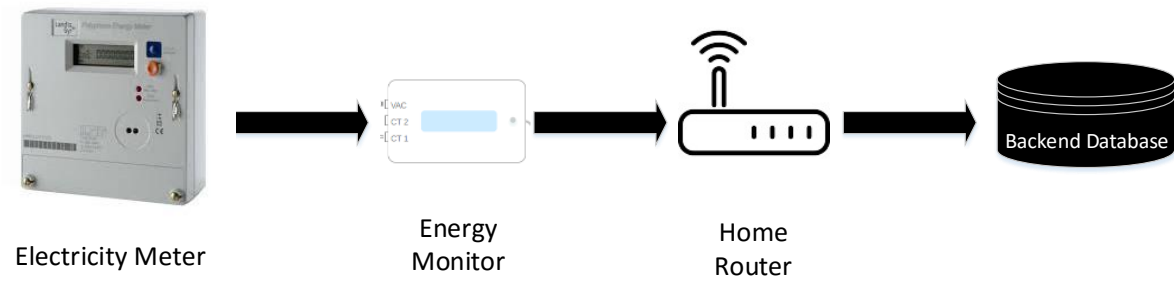

Figure 4. End to end data collection.

Increasing the reading frequencies facilitates the identification of induvial device utilisation this is demonstrated in Figure 5. The y-axis highlights the energy usage in Watts, while the $\mathrm{x}$-axis shows the reading time. 


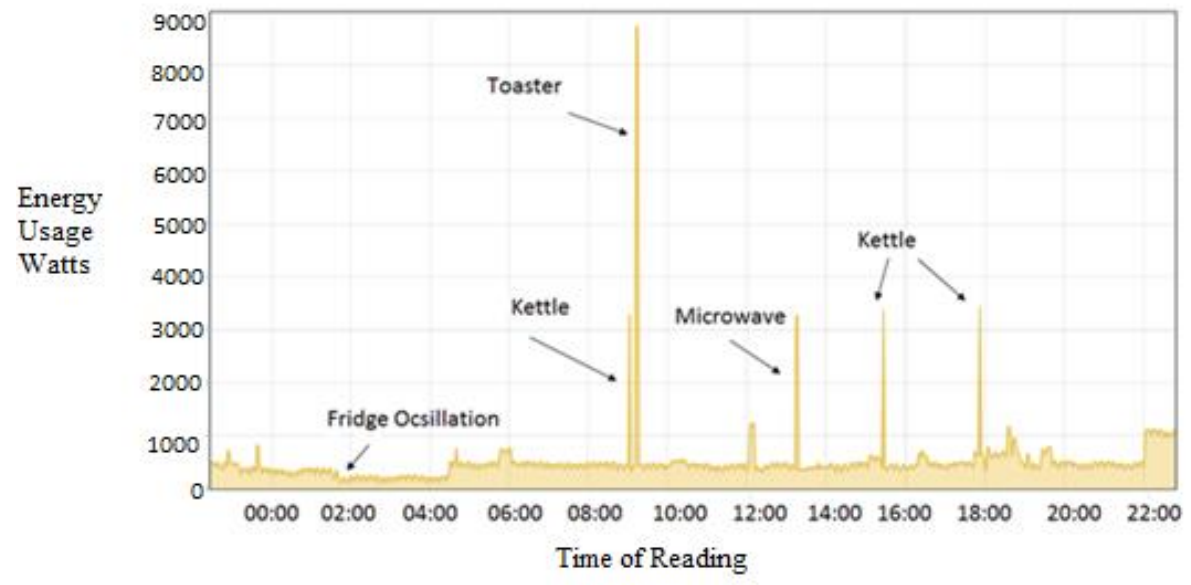

Figure 5. Real time energy readings.

Obtaining energy readings at 1 to 10 second intervals constructs individual energy signatures for each device. This is achieved by identifying the amount of energy being consumed, as demonstrated in Figure 6; where, as before, the y-axis displays energy usage in Watts and the $\mathrm{x}$-axis shows the reading time. This allows background noise from certain devices, such as the fridge oscillation, air conditioning and standby electricity usage, to be filtered out to leave clear usage signatures for important devices, such as a kettle, lights and cooking equipment. 


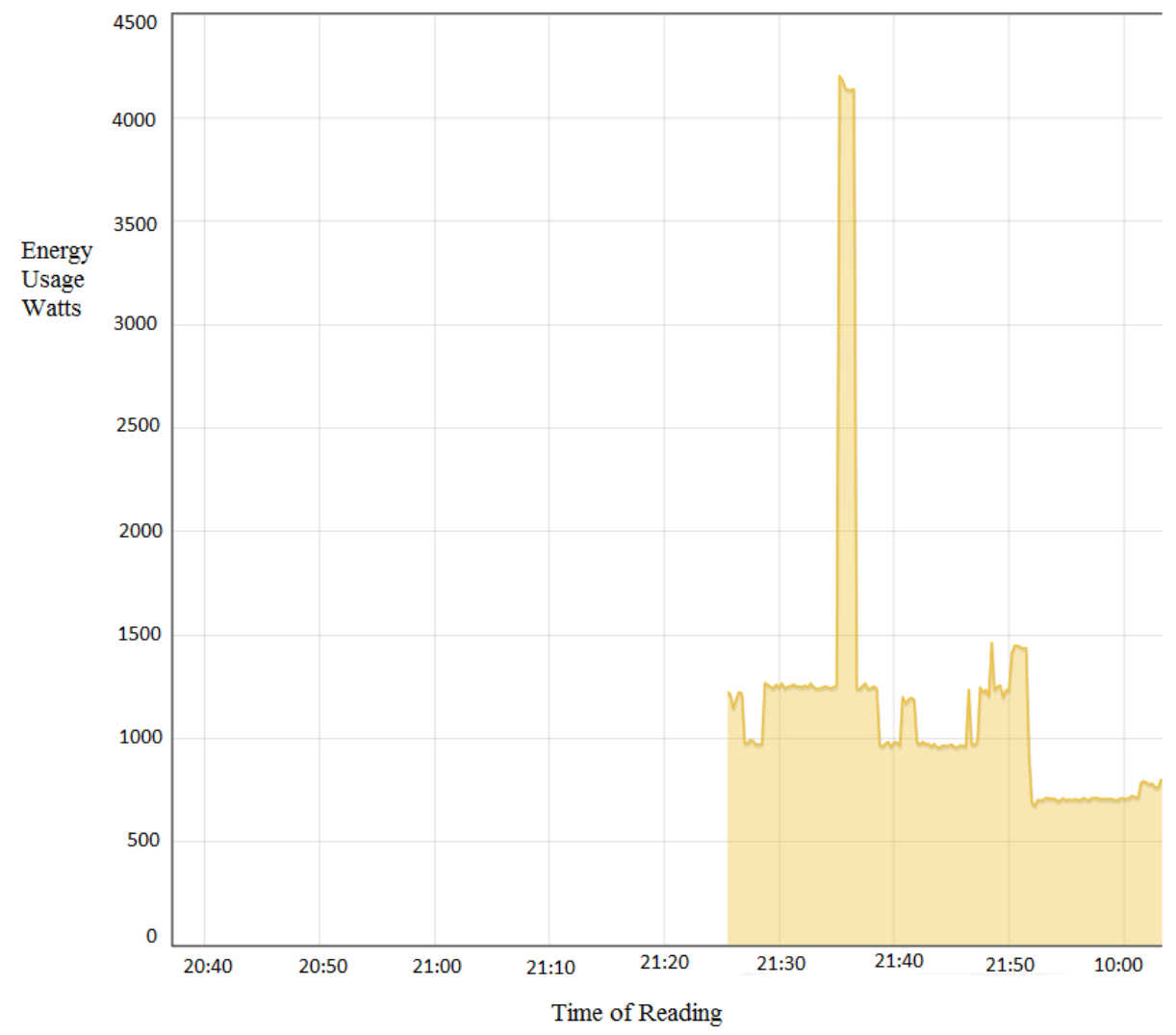

Figure 6. Energy signature for a kettle.

As previously highlighted, changes in sleep patterns are a common feature in several mental-health medical conditions. Being able to recognise changes and patterns in behaviour and, in particular, sleep disturbances, facilitates the prediction of future cognitive and non-cognitive changes. Often, dementia sufferers in hospital are admitted due to other poor health caused by other illnesses [19]. These are often caused by patient inactivity which ultimately leads to additional health complications. Detecting these behaviours early enables the prevention of additional complications.

\subsection{Methodology}

The data processing components of the system operates in two modes; firstly, the training mode, which involves collecting and processing energy usage data to train the data classifiers. Secondly, the prediction mode identifies both normal and abnormal behaviours using the trained classifiers from mode 1 .

Mode 1 - Training: When the system is in training mode normal and abnormal data is collected from the data store. Normal data refers to a patient's usual behavioural routines in a household. Abnormal data relates to a deviation from expected patterns 
of behaviour. Data features are extracted from the data set in order to train the classifiers to be able to detect abnormal patterns in a dataset.

Mode 2 -Prediction: Using the trained classifiers the system automatically detects both normal and abnormal patient behaviour in real-time. Where appropriate the system alerts the patients support network to a potential problem if detected.

In the first instance the system alerts the patient to check in, by performing specific device interaction. This reduces any possible false alarms and verifies that the patient requires no further assistance. The system identifies if interaction has taken place; if this is not the case an alert is communicated to a third-party health care practitioner as shown if figure 7 both of these modes of operation or subsequently detailed in this section.

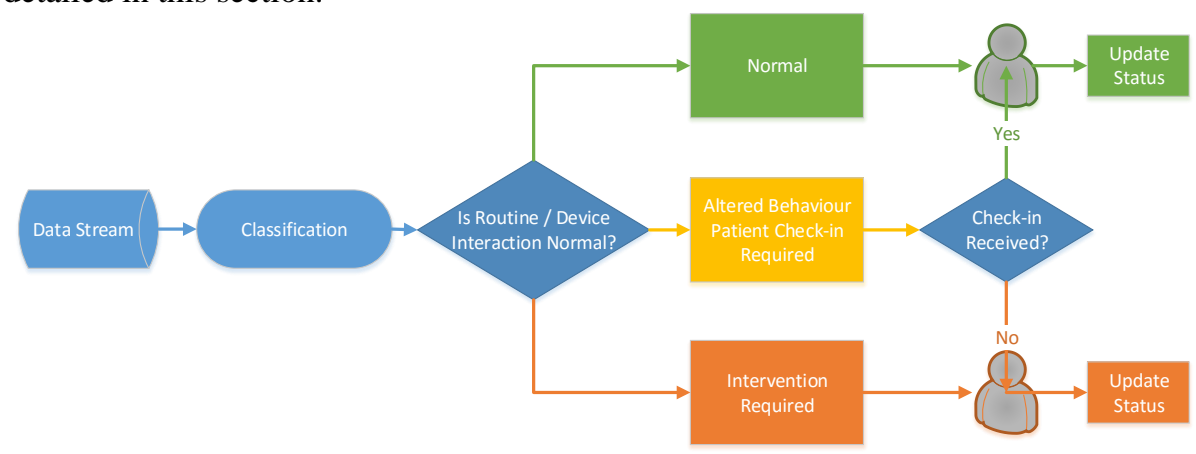

Figure 7. Mode two.

\section{Evaluation}

For the following analysis, one year's worth of energy usage readings for 8 different smart meter users was selected. The 8 consumers were selected as a sub group of the population as they accurately represent the population as a whole, this approach is more practical for the initial data analysis. Out of the 8 consumers selected 4 have normal readings and 4 have abnormal readings. The subjects with normal readings were classified as having no energy usage readings greater than 2 Kwh between the hours of 1:30 and 4:00 for the entire year period. Abnormal subjects were classified where they exceeded $2 \mathrm{Kwh}$ between the hours of 1:30 and 4:00 on 3 or more occasions in a one-year period. All households in the experiment have one occupant to ensure accurate results. Initially 7 features per consumer were derived for each 24-hour period totalling 8760 results for each of the following features: General supply min; general supply max; general supply median; general supply standard deviation; general supply mean; off peak max and off peak mean.

\subsection{Dimensionality Reduction}

In order to ascertain the optimum features and the greatest variance for the classification, Principle Component Analysis (PCA) is undertaken on the features created from the variables in the dataset (with each variable being a smart meter) this process is shown in Figure 8 during the feature selection step. General supply min, general supply max, general supply median, general supply standard deviation, general supply mean, off peak max and off peak mean, were devised from each 
variable to establish a total set of 28 features. Using this methodology helps to ensure the identification of the most useful features and a reduction of $67 \%$ in the number of features was obtained (left 9 out of the original 28).

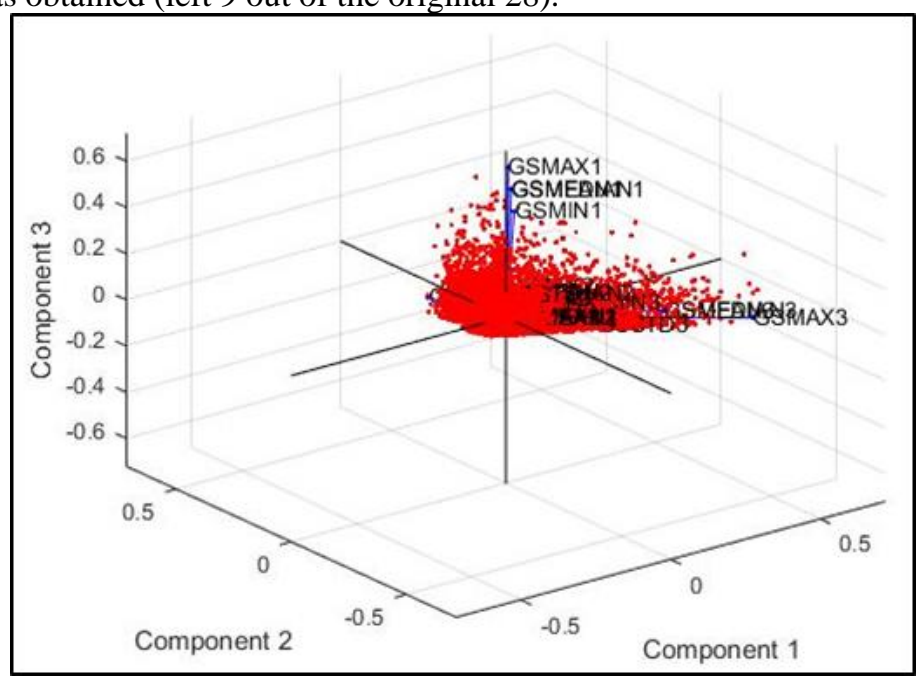

Figure 8. PCA Analysis.

\subsection{Neural Networks Classification}

In order to perform the classification of the data a selection of classifiers where used these include: back-propagation trained feed-forward neural network classifier (BPXNC), levenberg-marquardt trained feed-forward neural net classifier (LMNC), automatic neural network classifier (NEURC), radial basis function neural network classifier (RBNC), trainable linear perceptron classifier (PERLC), voted perception classifier (VPC) and the random neural network classifier (RNNC) [21].

Each of these classifiers where selected for their ability to learn how to recognise abnormal values in a dataset [22]. They also employ a supervised learning approach, which is a key part of the approach. The classifiers' performance is calculated using confusion matrices to assess the success of the classification or Area under the Curve (AUC), sensitivity, specificity and error [23]. Table 1 presents the confusion matrix for the NEURC classifier as an example.

Table 1. Confusion Matrix for NEURC

\begin{tabular}{|c|c|c|c|}
\hline \multirow{2}{*}{ True Labels } & \multicolumn{3}{|c|}{ Estimated Labels } \\
\hline & 1 & 2 & Totals \\
\hline 1 & 4133 & 246 & 4379 \\
\hline 2 & 107 & 4272 & 4379 \\
\hline Totals & 424- & 4518 & 8758 \\
\hline
\end{tabular}


The results are calculated mathematically, using the following formulae, where $T P$ refers to True Positive, $T N$ implies True Negative and $F P$ and $F N$ refer to False Positive and False Negative respectively.

$$
\begin{aligned}
& \text { Sensitivity }=T P /(T P+F N) \\
& \text { Specificity }=T N /(T N+F P)
\end{aligned}
$$

$$
\text { Accuracy }=(T P+T N) /(T P+F P+F N+T N)
$$

The NEURC is the most accurate; able to classify $95.96 \%$ of the data correctly with an error of 0.040 . For the NEURC classifier 4133 out of 4379 normal behaviours are correctly classified. The same classifier is able to assess 4272 out of 4379 abnormal behaviours are accurately classified on average. All of the results from the classification experiments are depicted in Table 2.

Table 2. Classification Results

\begin{tabular}{|c|c|c|c|c|}
\hline Classifiers & AUC (\%) & Sensitivity & Specificity & Error \\
\hline VPC & 84.81 & 0.844 & 0.851 & 0.1519 \\
\hline RNNC & 93.26 & 0.914 & 0.950 & 0.0674 \\
\hline PERLC & 64.01 & 0.301 & 0.978 & 0.3599 \\
\hline BPXNC & 89.50 & 0.887 & 0.902 & 0.105 \\
\hline LMNC & 92.57 & 0.898 & 0.953 & 0.074 \\
\hline NEURC & 95.96 & 0.943 & 0.975 & 0.040 \\
\hline RBNC & 93.33 & 0.898 & 0.968 & 0.107 \\
\hline
\end{tabular}

\subsection{Discussion}

It is clear from the results in Table 2, that the classifiers are able to detect accurately both the normal and abnormal behaviours in the data set. Figure 9 shows a visualisation of two features in a scatter plot. However this type of visualisation makes it challenging to see the division of the abnormal behaviour from the normal patterns. 


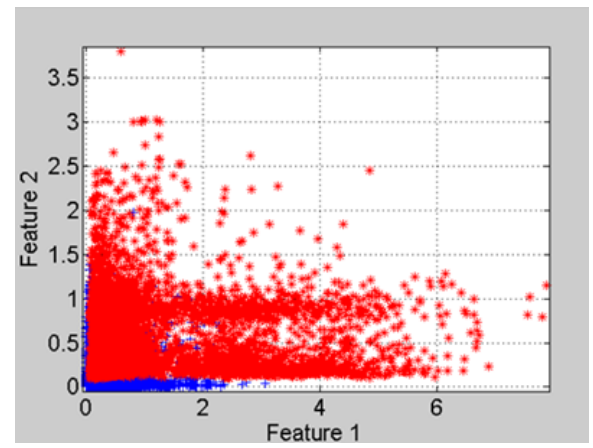

Figure 9. Visualisation of two features.

This division, can be made clearer through use of a Banana Plot, displayed in Figure 10, showing a stronger division in the data.

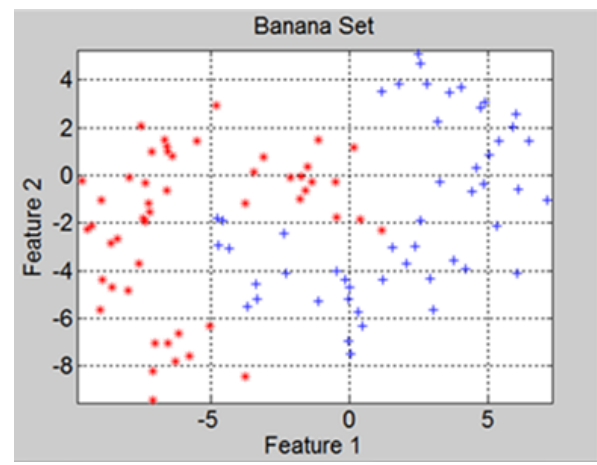

Figure 10. Banana Plot Highlighting division.

Figure 11 shows a visualisation if the NEURC classification process, overlaid on the Banana Plot; where the contour lines represent a conception of the data separation process during calculation.

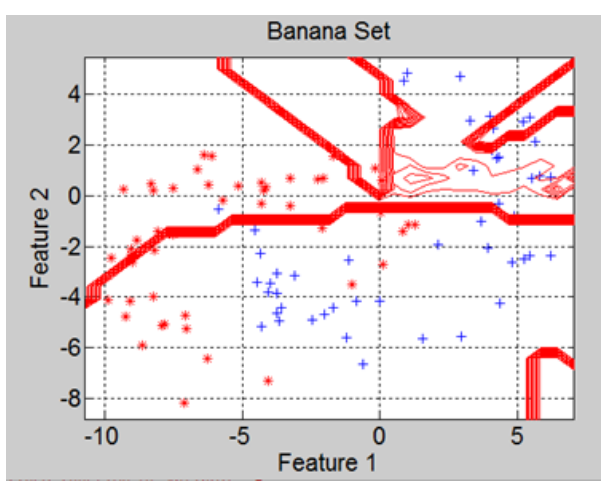

Figure 11. NEURC Classification Visualisation. 
Using the above techniques, support the findings that neural networks can be used to detect abnormal behaviour in smart meter datasets for health care monitoring. Using this approach, our system is able to perform an analysis of real-time datasets to detect when a user's behaviour is changing as a result of illness. The neurc classifier provides an accurate monitoring algorithm for monitoring people living with selflimiting conditions requiring an enable early intervention practice (EIP).

\section{Conclusion}

The implementation of smart meters and their associated infrastructures addresses many of the traditional constraints imposed by the current energy generation and distribution infrastructure. It allows detailed monitoring and consumer energy usage profiling. This leads to more efficient energy usage, planning and fault tolerance. Being able to collect and analyse sufficient amounts of usage data makes it possible to identify reoccurring patterns and trends in a patient's behaviour.

This can be utilised to address many problems, not just in the field of electricity and generation but also in health monitoring. In this paper, we have discussed the need to understand patient's behaviours based on their conditions and how these behaviours can be reflected in their energy usage. Identifying these changes and patterns facilitate the possibility for independent living and early intervention practice, reducing the demand for hospital care and enhanced resource planning.

The case study put forward in this research investigates the detection of sleep disturbances, which is one of the many key indicators of an onset or change in condition. We have demonstrated how this can be achieved by analysing smart meter data using our proposed methodology and by implementing neural network classifiers to detect abnormal energy usage.

\section{References}

[1] Department of Health, 'Report Long-term conditions compendium of Information: 3rd edition' (2013), Published to DH website.

[2] Margaret Chan, 'Dementia A public health priority, World Health Organization' (2012) http://www.globalaging.org/agingwatch/Articles/Dementia\%20a\%20public\%20 health\%20priority.pdf (accessed 12/02/2016).

[3] Mental Health Foundation, 'The Fundamental Facts The latest facts and figures on mental health', [online] http://www.mentalhealth.org.uk/content/assets/PDF/publications/fundamental f acts 2007.pdf?view=Standard (accessed 18/01/2016). 
[4] Prince, M, Knapp, M, Guerchet, M, McCrone, P, Prina, M, Comas-Herrera, A, Wittenberg, R, Adelaja, B, Hu, B, King, D, Rehill, A \& Salimkumar, D 2014, Dementia UK: Second Edition - Overview. Alzheimer's Society.

[5] Paul McCrone, Sujith Dhanasiri, Anita Patel, Martin Knapp and Simon LawtonSmith, PAYING THE PRICE The cost of mental health care in England to 2026, King's Fund.

[6] Mental Health Network NHS CONFEDERATION, Early intervention in psychosis services, 2011.

[7] M. Anas, N. Javaid, A. Mahmood, S. M. Raza, U. Qasim, Z. A. Khan 'Minimizing Electricity Theft using Smart Meters in AMI' Seventh International Conference on P2P, Parallel, Grid, Cloud and Internet Computing, 2012.

[8] M. Popa. 'Data Collecting from Smart Meters in an Advanced Metering Infrastructure' INES 2011 15th International Conference on Intelligent Engineering Systems, June 2011, pp. 137 - 142.

[9] Coalton Bennett and Darren Highfill. Networking AMI Smart Meters, IEEE Energy2030, November 2008.

[10] Andr'es Molina-Markham, Prashant Shenoy, Kevin Fu, Emmanuel Cecchet, and David Irwin, (2010) 'Private Memoirs of a Smart Meter' BuildSys '10 Proceedings of the 2nd ACM Workshop on Embedded Sensing Systems for Energy-Efficiency in Building, pp. 61-66.

[11] Lingfeng Wang, Devabhaktuni, V., Gudi, N., 'Smart Meters for Power Grid Challenges, Issues, Advantages and Status' 2011 IEEE/PES Power Systems Conference and Exposition (PSCE), pp. 1-7, March 2011.

[12] Francesco Benzi, Norma Anglani, Ezio Bassi and Lucia Frosini Electricity Smart Meters Interfacing the Households, IEE transactions on Industrial Electronics, Vol 58, No10, VOL. 58, NO. 10, 2011.

[13] Eoghan McKenna, Ian Richardson, Murray Thomson, (2012) 'Smart meter data: Balancing consumer privacy concerns with legitimate applications' Energy Policy, VOL.41, pp. 807-814.

[14] Vojdani (2008) 'A Smart integration' IEEE Power \& Energy Magazine, vol71 issue 9 , pp. 71-79.

[15] Department of Energy and Climate Change 'Smart Meters, Smart Data, Smart Growth' (2015) [online] https://www.gov.uk/government/publications/smartmeters-smart-data-smart-growth (accessed 22/02/2016). 
[16] Michael S. Mega, Jeffrey L. Cummings, Tara Fiorello, Jeffrey Gornbein 'The spectrum of behavioural changes in Alzheimer's disease' NEUROLOGY, 1996, VOL.46, pp. 130-135.

[17] Madhukar H Trivedi, (2004) 'The link between depression and physical symptoms' Primary care companion to the Journal of clinical psychiatry, VOL.6, pp. 12-16.

[18] Early Psychosis [online] http://www.earlypsychosis.ca/pages/curious/warning-signs-of-psychosis] accessed October 2015 (accessed 14/03/2016).

[19] Sandra Selikson, Karla Damus, David Hameramn (2015) 'Risk Factors Associated with Immobility' Journal of the American Geriatrics Society.

[20] Chiara Baglionia, Gemma Battaglieseb, Bernd Feigea, Kai Spiegelhaldera, Christoph Nissena, Ulrich Voderholzera, c, Caterina Lombardob, Dieter Riemanna, Insomnia as a predictor of depression: A meta-analytic evaluation of longitudinal epidemiological studies, Journal of Affective Disorders, 2011.

[21] Hyong Jin Cho, Helen Lavretsky, Richard Olmstead, Myron J. Levin, Michael N. Oxman, Michael R. Irwin, Sleep Disturbance and Depression Recurrence in Community-Dwelling Older Adults: A Prospective Study, THE AMERICAN JOURNAL OF PSYCHIATRY, December 2008.

[22] N. Marom, L. Rokach, and A. Shmilovici, Using the Confusion Matrix for Improving Ensemble Classifiers, Proceedings of the Twenty-Sixth IEEE Convention of Electrical and Electronics Engineers in Israel, pp. 000555000559, 2010.

[23] Ahmed J. Aljaaf, Dhiya Al-Jumeily, Abir J. Hussain, Paul Fergus, Mohammed Al-Jumaily and Naeem Radi, Applied Machine Learning Classifiers for Medical Applications: Clarifying the behavioural patterns using a variety of datasets, Systems, Signals and Image Processing (IWSSIP), 2015 International Conference, September 2015. 